\title{
Isolated Severe Bilateral Bronchomalacia: Correspondence
}

\author{
Vijayasekaran Dharmalingam • Shanthi Ramesh
}

Received: 20 August 2014 / Accepted: 25 September 2014 / Published online: 6 November 2014

(C) Dr. K C Chaudhuri Foundation 2014

To the Editor: We read the article on 'Isolated Severe Bilateral Bronchomalacia' and would like to seek few clarifications and also share some useful information [1].

Airway malacia is increasingly diagnosed after the widespread use of fiberoptic bronchoscopy, especially in infants. In centers where facility for fiberoptic bronchoscopy is available, it has been documented that, airway malacia is found to be one of the causes for infantile wheeze especially if the symptoms occur at 4 to $8 \mathrm{wk}$ after birth and persist without signs of respiratory infection [2].

Though authors consider airway malacia as an uncommon condition, primary airway malacia is not a rare entity in the general population with an estimated incidence of 1 in 2,100 children [3].

In our experience, bilateral bronchomalacia appears as fish mouth appearance under bronchoscopic vision due to collapsibility of both the bronchi but the authors have reported collapsibility of bronchi along with prominence of blood vessels in the left main bronchus, which raises the issue whether any associated anomalies were present on that side causing external compression.

Managing a child with bilateral bronchomalacia presenting with repeated acute life threatening episodes (ALTE), is difficult. Multilobar infiltrates and pulmonary hypertension due to persistent hypoxemia were the presenting features in our experience.

In the absence of definitive management protocols for airway malacia we suggest that medical approach should comprise of a comprehensive strategy incorporating the following important principles like (a) treatment of the underly-

V. Dharmalingam $\cdot$ S. Ramesh $(\bowtie)$

Department of Pediatrics, Sree Balaji Medical College Hospital, Chennai 600010, India

e-mail: drshanthiramesh@gmail.com ing infection, (b) avoiding nebulization with beta 2 agonist (as beta agonists reduce the muscle tone and make the bronchi more compliant), (c) continuous positive airway pressure in children presenting with hypoxemia (useful to open the collapsing bronchi), (d) chest physiotherapy (to clear the backlog of retained secretions in the distal airway) and (e) use of nebulized ipratropium for bronchodilation [4].

Though surgical approaches currently utilised for management of intrinsic malacia have not been fully validated, aortopexy is found to be useful in older children with tracheomalacia [2]. The authors have discussed the airwaystent related complications and cautioned that such interventions should be discouraged in the pediatric age group.

They have again made a valid recommendation that if an infant has persistent wheezing despite adequate therapy, airway malacia should be considered in the differential diagnosis and making an early diagnosis of airway malacia by fiberoptic bronchoscopy may avoid repeated hospitalizations and unnecessary treatment.

Conflict of Interest None.

Source of Funding None.

\section{References}

1. Saikia B, Sharma PK, Sharma R, Gagneja V, Khilnani P. Isolated severe bilateral bronchomalacia. Indian J Pediatr. 2014;81:707-8.

2. Carden KA. Tracheomalacia and tracheobronchomalacia in children and adults -an in-depth review. Chest. 2005;127:984-1005.

3. Boogaard R, Huijsmans SH, Pijnenburg MW, Tiddens HA, de Jongste JC, Merkus PJ. Tracheomalacia and bronchomalacia in children: incidence and patient characteristics. Chest. 2005;128:3391-7.

4. Vijayasekaran D, Kalpana S, Vivekanandan VE. Tracheobronchomalacia presenting as infantile wheeze. Indian Pediatr. 2011;48:903-4. 